\title{
The prevalence and risk factors of young male schizophrenics with non-alcoholic fatty liver disease
}

This article was published in the following Dove Press journal:

Neuropsychiatric Disease and Treatment

12 June 2017

Number of times this article has been viewed

\author{
Jing Yan ${ }^{1, *}$ \\ Chengye Hou ${ }^{1,2, *}$ \\ Ying Liang' \\ 'National Clinical Research Center \\ for Mental Disorders, Peking \\ University Sixth Hospital, Institute \\ of Mental Health, Key Laboratory of \\ Mental Health, Ministry of Health, \\ Peking University, Beijing, China; \\ ${ }^{2}$ Liaoning Province Demobilized \\ Soldiers Kangning Hospital, \\ Huludao, China \\ *These authors contributed equally \\ to this work
}

Correspondence: Ying Liang National Clinical Research Center for Mental Disorders, Peking University Sixth Hospital, Institute of Mental Health, Key Laboratory of Mental Health, Ministry of Health, Peking University, 5 I huayuanbei road, Haidian I00191, Beijing, China Email liangying1980@bjmu.edu.cn
Objective: In this study, we compared the prevalence rate of non-alcoholic fatty liver disease (NAFLD) between young males with schizophrenia and the general young males as the control group, and we also investigated the risk factors of NAFLD in young males with schizophrenia.

Materials and methods: This is a large cross-sectional study consisting of the study group and parallel control group. The study group comprises 202 cases of young males with schizophrenia recruited from Liaoning province Demobilized Soldiers Kangning Hospital, and 149 healthy young males were recruited as the control group. The clinical evaluation included Positive and Negative Syndrome Scale (PANSS), basic information, disease-related information, and physical examination information. The laboratory tests included fasting blood glucose, total cholesterol, triglyceride, alanine aminotransferase (ALT), and aspartate aminotransferase (AST). The physical examination included liver ultrasonography.

Results: No statistical difference was observed between the study and control groups regarding

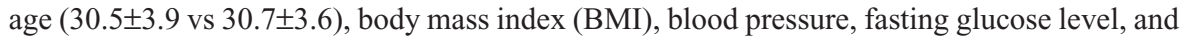
total cholesterol. NAFLD was observed in both the study and control groups. The prevalence of NAFLD was $49.5 \%(100 / 202)$ in the study group, and 20.1\% (30/149) in the control group. The study group was further divided into NAFLD group and non-NAFLD group. There were significant differences in medication combination, drug dosage, negative factor score in PANSS (14.1 \pm 4.0 vs $12.7 \pm 4.5$ ), BMI, fasting glucose level, total cholesterol, triglyceride, ALT, and AST between NAFLD group and non-NAFLD group. According to the results of the multiplefactor analysis, the onset of NAFLD among young males with schizophrenia was significantly correlated with the following factors: triglyceride, BMI, medication combination, drug dosage, and negative factor score in PANSS.

Conclusion: The prevalence of NAFLD in the study group was significantly higher than that in the control group. Multivariate analysis indicated that, triglyceride, BMI, medication combination, drug dosage, and negative factor score in PANSS were significantly related to NAFLD in patients with schizophrenia.

Keywords: schizophrenia, NAFLD, risk, risk factors, male

\section{Introduction}

Schizophrenia is a complex and severe mental disorder which generally appears in late adolescence or early adulthood and affects approximately $1 \%$ of the population. As schizophrenia is a chronic disease, social functioning will be impaired continuously as the condition worsens. Since the middle of the last century, antipsychotics, which have been widely used in the clinical setting, play an important role in increasing the remission rate of psychiatric symptoms and improving the social functioning 
of patients. However, the dangers of long-term use of antipsychotic drugs have been widely acknowledged. For instance, the risk of common medical comorbidities such as obesity, hypertension, hyperlipidemia, and diabetes mellitus is significantly higher than that in the general population. ${ }^{1}$ Schizophrenia itself may be associated with lack of exercise and detrimental dietary habits; negative symptoms and lower social functioning are more common in these patients, especially in males. ${ }^{2}$ Both metabolic comorbidity and unhealthy lifestyle habits are high-risk factors of non-alcoholic fatty liver disease (NAFLD). ${ }^{3}$

NAFLD, a kind of chronic liver disease, is the second most common liver disease after viral hepatitis. Among NAFLD cases, $5 \%-10 \%$ are reported to develop cirrhosis, and $1 \%-2 \%$ to develop hepatocellular cancer. ${ }^{4}$ Also, NAFLD increases the risk of cardiovascular and cerebrovascular diseases. ${ }^{5} \mathrm{With}$ the change in diet and lifestyle, the prevalence of NAFLD is increasing year by year (especially in males). ${ }^{6}$ The age at onset of NAFLD in schizophrenia tends to be younger. ${ }^{7}$

To our knowledge, a few studies have been performed to analyze the potential development of NAFLD in individuals with schizophrenia. The previous study showed that the potential for NAFLD in first episode schizophrenia spectrum patients was increased after receiving antipsychotic medication for 3 years. ${ }^{8}$ Remarkable negative symptoms are common in the male patient with schizophrenia, which generally appear in early adulthood. In addition, the age at onset of NAFLD becomes younger. Based on these studies, we speculated that the prevalence rate of NAFLD in schizophrenia is higher among males. In order to compare the prevalence rate of NAFLD between young male schizophrenics and healthy young males, and investigate the risk factors of NAFLD in young males with schizophrenia, we performed a large sample cross-sectional survey among young male schizophrenics with NAFLD.

\section{Materials and methods Subjects}

In this large sample cross-sectional study which started in May 2015, we screened 687 schizophrenics who met the diagnostic criteria for schizophrenia according to ICD- $10^{9}$ in Liaoning Demobilized Soldiers Hospital ward. Inclusion criteria were: 1) aged 18-35 years; 2) male gender; 3) steadily taking antipsychotic drugs for the last 1 month; 4) no history of alcohol abuse; 5) not taking liver-protecting drugs; 6) free of certain diseases causing fatty liver such as viral hepatitis, total parenteral nutrition (TPN), and hepatolenticular degeneration (HLD). Finally, 202 cases were qualified in our study. Healthy controls of this study were recruited from the staff in the same hospital. Inclusion criteria for healthy controls were as follows: 1) aged 18-35 years; 2) male gender; 3) no history of alcohol abuse (or ethyl alcohol content $<140 \mathrm{~g} /$ week); 4) not taking liver-protecting drugs; 5) free of certain diseases causing fatty liver such as viral hepatitis, TPN, and HLD. Finally, 149 people were qualified.

The study protocol and informed consent were in accordance with the Guidelines for Good Clinical Practice of the International Conference on Harmonisation (ICH-GCP), ${ }^{10}$ which was approved by the Ethics Committee of Peking University, Institute of Mental Health. Prior to the enrollment, signed written informed consent was obtained from the subjects or their legal guardians for this study.

\section{Clinical assessment}

Positive and Negative Syndrome Scale (PANSS) was used to assess the severity of disease. ${ }^{11}$ A questionnaire was used to collect the general information and disease conditions including age, gender, date of birth, education level, ethnicity, marital status, age of onset, disease duration, previous disease history, medication history, smoking history, and current medication list. In addition, the current dose of antipsychotic was converted to the equivalent dose of chlorpromazine. ${ }^{12}$

Physical examination was performed and recorded by professional physicians. Height and weight data were measured by an automatic measuring instrument. Body mass index (BMI) was derived from weight divided by the square of the height. Blood pressure was measured by an automatic electronic sphygmomanometer (systolic pressure and diastolic pressure).

\section{Laboratory tests}

Venous blood samples were collected by nurses from the subjects at 6-7 am after 8-12-hour fasting. Biochemical tests (fasting blood glucose, total cholesterol, triglyceride, alanine aminotransferase [ALT], and aspartate aminotransferase [AST]) were performed by a senior technician using Toshiba TBA-120FR (Tokyo, Japan).

\section{Physical examination}

After 8-hour fasting, the ultrasound test of liver function was performed by a senior technician using $\alpha-7$ color ultrasonography. The diagnostic criteria of NAFLD were confirmed by the diagnostic criteria for NAFLD classification revised by Chinese Academy of Liver disease in 2010. ${ }^{13}$

\section{Statistical analysis}

SPSS 17.0 was used for the data analysis. Descriptive analysis was used for the general demographic data and clinical features. Continuous variables were shown as mean $\pm \mathrm{SD}$, and classification variables were shown as frequency and 
percentage. Patients were further divided into NAFLD group and non-NAFLD group. Single factor analysis was used to analyze the data of NAFLD group and non-NAFLD group. Continuous variables were compared by independent-samples $t$-test and rank-sum test. Categorical data were compared with chi-square. Logistic regression analysis was used to assess the correlation between effects of different risk factors and NAFLD. Statistical significance was defined as $P<0.05$.

\section{Results}

\section{Comparison of the NAFLD risk}

The mean age showed no significant difference between the study group (30.5 \pm 3.9$)$ and control group (30.7 \pm 3.6$)$. The prevalence of NAFLD was $49.5 \%(100 / 202)$ in study group and $20.1 \%(30 / 149)$ in control group. In control and study groups, there were significant differences in the data of increased triglyceride cases and the increased numerical value of triglyceride $(P<0.05)$ (Table 1$)$.

\section{Risk factors for NAFLD}

In this study, NAFLD was diagnosed according to the "Guidelines for the diagnosis and treatment of nonalcoholic fatty liver disease", promulgated by the fatty liver and alcoholic liver disease group, Chinese society of Hepatology,

Table I Demographic and clinical data of the study and control groups

\begin{tabular}{|c|c|c|c|c|c|c|}
\hline & \multicolumn{2}{|c|}{$\begin{array}{l}\text { Study } \\
\text { group } \\
(n=202) \\
\end{array}$} & \multicolumn{2}{|c|}{$\begin{array}{l}\text { Control } \\
\text { group } \\
(n=\mid 49)\end{array}$} & \multirow[t]{2}{*}{$\chi^{2}$} & \multirow[t]{2}{*}{$P$-value } \\
\hline & $\mathbf{n}$ & $\%$ & $\mathbf{n}$ & $\%$ & & \\
\hline Smoking (n) & 40 & 19.8 & 24 & 16.1 & 0.79 & 0.404 \\
\hline $\begin{array}{l}\text { Increased fasting glucose } \\
\text { level }\end{array}$ & 33 & 16.3 & 14 & 9.4 & 3.56 & 0.080 \\
\hline Increased total cholesterol & 16 & 7.9 & 18 & 12.1 & 1.70 & 0.206 \\
\hline Increased triglyceride & 108 & 53.5 & 61 & 40.9 & 5.39 & 0.023 \\
\hline Increased ALT & 42 & 20.8 & 27 & 18.1 & 0.39 & 0.588 \\
\hline \multirow[t]{2}{*}{ Increased AST } & 25 & 12.4 & II & 7.4 & 2.32 & 0.155 \\
\hline & Mean & SD & Mean & SD & $\mathbf{T}$ & $P$-value \\
\hline Age (years) & 30.5 & 3.9 & 30.7 & 3.6 & -0.56 & 0.574 \\
\hline BMI & 23.0 & 3.5 & 23.2 & 3.1 & -0.50 & 0.615 \\
\hline Systolic blood pressure & 126.7 & 9.5 & 124.9 & 8.1 & 1.84 & 0.067 \\
\hline Diastolic blood pressure & 73.7 & 6.4 & 72.5 & 5.4 & 1.87 & 0.062 \\
\hline $\begin{array}{l}\text { Fasting glucose level } \\
(\mathrm{mmol} / \mathrm{L})\end{array}$ & 5.0 & 1.2 & 5.1 & 1.2 & 1.11 & 0.267 \\
\hline $\begin{array}{l}\text { Total cholesterol } \\
(\mathrm{mmol} / \mathrm{L})\end{array}$ & 5.0 & 1.2 & 5.1 & 1.2 & -0.53 & 0.594 \\
\hline Triglyceride $(\mathrm{mmol} / \mathrm{L})$ & 2.3 & 1.6 & 2.0 & 1.6 & 2.13 & 0.034 \\
\hline $\operatorname{ALT}(\mathrm{u} / \mathrm{L})$ & 38.7 & 23.0 & 38.2 & 23.1 & 0.19 & 0.852 \\
\hline AST (u/L) & 27.6 & 12.4 & 26.6 & 10.7 & 0.81 & 0.418 \\
\hline $\operatorname{ALP}(u / L)$ & 64.4 & 16.5 & 62.2 & 14.4 & 1.29 & 0.199 \\
\hline GGT (u/L) & 49.8 & 29.5 & 47.4 & 36.8 & 0.69 & 0.493 \\
\hline
\end{tabular}

Abbreviations: ALT, alanine aminotransferase; ALP, alkaline phosphatase; AST, aspartate aminotransferase; BMI, body mass index; GGT, gamma-glutamyltransferase.
Table 2 Comparison of general population information and clinical data between the NAFLD group and non-NAFLD group

\begin{tabular}{|c|c|c|c|c|c|c|c|}
\hline & \multicolumn{2}{|c|}{$\begin{array}{l}\text { NAFLD } \\
\text { group } \\
(\mathrm{n}=\mid 00)\end{array}$} & \multicolumn{2}{|c|}{$\begin{array}{l}\text { Non- } \\
\text { NAFLD } \\
\text { group } \\
(\mathrm{n}=102)\end{array}$} & \multirow[t]{2}{*}{$\chi^{2}$} & \multirow[t]{2}{*}{$d f$} & \multirow[t]{2}{*}{$P$-value } \\
\hline & $\mathrm{n}$ & $\%$ & $\mathbf{n}$ & $\%$ & & & \\
\hline History of smoking & 22 & 22.0 & 18 & 17.6 & 0.6 & 1 & 0.483 \\
\hline \multicolumn{8}{|l|}{ Antipsychotics } \\
\hline Medication combination & 63 & 63.0 & 37 & 36.3 & 14.4 & I & 0.000 \\
\hline Drug doses (CPZ) & & & & & 15.0 & 2 & 0.001 \\
\hline$<300 \mathrm{mg}$ & 5 & 5.0 & 18 & 17.6 & & & \\
\hline $300-600 \mathrm{mg}$ & 29 & 26.0 & 39 & 38.2 & & & \\
\hline \multirow[t]{2}{*}{$>600 \mathrm{mg}$} & 69 & 69.0 & 45 & 44.1 & & & \\
\hline & Mean & SD & Mean & SD & $\overline{\mathbf{T}}$ & $d f$ & $P$-value \\
\hline Age (years) & 30.5 & 3.8 & 30.5 & 4.0 & 0.09 & 200 & 0.929 \\
\hline Disease duration (year) & 7.1 & 4.0 & 5.6 & 5.3 & 2.31 & 200 & 0.022 \\
\hline PANSS & 49.5 & 11.4 & 50.3 & 14.1 & -0.48 & 200 & 0.629 \\
\hline PANSS positive factors & 11.9 & 4.7 & 13.1 & 5.5 & -1.62 & 200 & 0.107 \\
\hline PANSS negative factors & 14.1 & 4.0 & 12.7 & 4.5 & 2.35 & 200 & 0.020 \\
\hline BMI & 24.1 & 3.9 & 21.9 & 3.7 & 4.52 & 200 & 0.000 \\
\hline Systolic pressure & 126.8 & 9.6 & 126.6 & 9.4 & 0.17 & 200 & 0.863 \\
\hline Diastolic pressure & 73.5 & 6.1 & 74.0 & 6.7 & -0.52 & 200 & 0.602 \\
\hline Fasting blood glucose & 5.3 & 1.4 & 4.7 & 0.9 & 3.51 & 200 & 0.001 \\
\hline Total cholesterol & 5.2 & 1.3 & 4.9 & I.I & 2.13 & 200 & 0.034 \\
\hline Triglyceride & 2.8 & 1.5 & 1.8 & 1.4 & 5.10 & 200 & 0.000 \\
\hline ALT & 44.3 & 24.2 & 33.2 & 20.4 & 3.54 & 200 & 0.001 \\
\hline AST & 30.9 & 13.8 & 24.4 & 9.8 & 3.86 & 200 & 0.000 \\
\hline ALP & 64.9 & 16.9 & 63.8 & 16.1 & 0.45 & 200 & 0.654 \\
\hline GGT & 53.7 & 20.9 & 46.0 & 35.6 & 0.86 & 200 & 0.065 \\
\hline
\end{tabular}

Abbreviations: ALT, alanine aminotransferase; ALP, alkaline phosphatase; AST, aspartate aminotransferase; GGT, gamma-glutamyltransferase; NAFLD, nonalcoholic fatty liver disease; CPZ, chlorpromazine; PANSS, Positive and Negative Syndrome Scale; BMI, body mass index.

and Chinese Medical Association. ${ }^{13}$ Patients were divided into NAFLD group and non-NAFLD group. The following data were significantly different between the two groups: medication combination, drug dose, negative factor score in PANSS, BMI, fasting blood glucose, total cholesterol, triglyceride, ALT, and AST (Table 2).

The fit of the overall logistic regression model was 0.388. Multivariate analysis indicated that triglyceride, BMI, medication combination, dose, and negative factor score in PANSS were significantly related to NAFLD (Table 3 ).

Table 3 Multivariate analysis of risk factors for NAFLD

\begin{tabular}{lllllll}
\hline Factor & B & SE & Wald test & P-value & OR & $\mathbf{9 5 \% ~ C l ~}$ \\
\hline Triglyceride & 0.36 & 0.13 & 7.39 & 0.007 & 1.44 & $\mathrm{I} .1 \mathrm{I}-\mathrm{I} .87$ \\
BMI & 0.15 & 0.05 & 7.30 & 0.007 & 1.16 & $\mathrm{I} .04-1.29$ \\
$\begin{array}{l}\text { Medication } \\
\text { combination }\end{array}$ & 0.83 & 0.34 & 5.84 & 0.016 & 2.90 & $\mathrm{I} .17-4.48$ \\
$\begin{array}{l}\text { Drug doses } \\
\text { PANSS }\end{array}$ & 0.80 & 0.26 & 9.62 & 0.002 & 2.36 & $\mathrm{I} .35-3.72$ \\
negative factors & 0.09 & 0.04 & 5.05 & 0.025 & 1.09 & $\mathrm{I} .0 \mathrm{I}-\mathrm{I} .18$
\end{tabular}

Abbreviations: NAFLD, non-alcoholic fatty liver disease; BMI, body mass index; PANSS, Positive and Negative Syndrome Scale. 


\section{Discussion}

To our knowledge, in China, this is the first study which investigates the prevalence of NAFLD in young males with schizophrenia, in which the study group was the same age and gender as the controls. A recent national epidemiological survey showed that the NAFLD prevalence in Chinese adults was $20.86 \%,{ }^{14}$ which was lower than that in Western countries $(30 \%) .{ }^{15}$ One possible factor is that the Western lifestyle and dietary habits were more likely to lead to obesity and insulin resistance. The peak age of NAFLD onset is during middle age. NAFLD has not been studied in the young and middle-aged population until now. This study first reported the prevalence of NAFLD in young males, and the result showed that the prevalence was equal to that in adults (20.1\%). The results suggest that the lifestyle of young men in China has changed, and the age of NAFLD onset tends to be younger. ${ }^{7}$

Our study found that the prevalence of NAFLD in young male patients with schizophrenia $(49.5 \%)$ is much higher than that in the general population, and the age of NAFLD onset in patients with schizophrenia is remarkably younger. Besides, 5\%-10\% of NAFLD patients are reported to develop cirrhosis, and 1\%-2\% are reported to develop hepatocellular cancer. ${ }^{4}$ In addition, NAFLD is an independent risk factor for atherosclerosis. The presence of NAFLD leads to an increased risk of endothelial dysfunction, ${ }^{16}$ and further to cardiovascular disease and cerebrovascular diseases. The presence of NAFLD will exacerbate the progression of physical diseases such as cirrhosis, hepatic carcinoma, cardiovascular disease, and cerebrovascular diseases. NAFLD will seriously affect patients' quality of life and life span, it will also lead to a heavy burden for patients' families. Based on these findings, much more attention should be paid to young male schizophrenic patients with NAFLD.

NAFLD is a clinical syndrome characterized by hepatocellular macrovesicular steatosis and necrosis, inflammatory cell infiltration, and fat accumulation. The main mechanisms of steatosis are the fat accumulation and disturbed lipid metabolism homeostasis in the liver. NAFLD is related to insulin resistance, transcription factors of lipogenesis, and gene polymorphism of triglyceride transfer protein gene.

Our study investigated the risk factors of NAFLD in young male patients with schizophrenia. A recent study showed that the potential for NAFLD in schizophrenia patients receiving antipsychotic medication is higher. But this study examined the incidence of NAFLD using a randomized design in mostly middle-aged schizophrenia patients, and just looked at the effects of specific antipsychotics on Western people. ${ }^{8}$ In our study, we focused on young male patients with schizophrenia and investigated risk factors other than antipsychotics.

This study showed that a large number of schizophrenia patients diagnosed with NAFLD are more likely to use combination therapy with a higher dose of antipsychotic drugs, which has not been reported in previous studies. It should be noted that larger doses should be used in combination therapy, but that more prominent adverse reactions would appear. ${ }^{17}$ In particular, it has been well-known that antipsychotics will cause side effects such as weight gain, obesity, and metabolic syndrome. ${ }^{1}$ Atypical antipsychotics have an effect on aminotransferase elevation. In addition, weight gain caused by atypical antipsychotics could also lead to NAFLD and steatohepatitis through mild aminotransferase elevation. ${ }^{18}$ Compared with single antipsychotic therapy, a multiple antipsychotics combination can increase the risk of metabolic syndrome and insulin resistance. ${ }^{19,20}$ The side effects, as mentioned previously, probably result from the blockage of 5-HT2c, H1, and M1 receptor by antipsychotic drugs. ${ }^{21}$ In addition, antipsychotics can inhibit the uptake of glucose by tissues directly, through blocking glucose transporters in the cell membrane. ${ }^{22}$ In addition, antipsychotics can promote the production of triglyceride and inhibit the metabolism of triglyceride. ${ }^{18}$ A recent study revealed that the prevalence of NAFLD increased during antipsychotic drug treatment. ${ }^{6}$ In the previous study, elevated blood-lipid and glucose levels were observed in the early course of antipsychotic drug treatment, and the incidence of disease will increase with the prolongation of the course. ${ }^{23}$ Thus, psychiatrists should pay more attention to the risk of NAFLD in patients with high-dose combination therapy, and early prevention and treatment are necessary and important for patients.

Our study showed for the first time, that negative symptoms are more serious in young male schizophrenic patients with NAFLD. The negative symptoms include anhedonia, poor attention, low motivation, apathy, and social withdrawal. Lack of exercise and negative lifestyles are common in patients with negative symptoms,${ }^{24}$ which increase the risk of dyslipidemia, obesity, insulin resistance, and diabetes (most of these are high-risk factors of NAFLD). ${ }^{14}$ Thus, we should focus on the negative symptoms in patients and offer a proper treatment to decrease the risk of NAFLD.

In this study, we found that high BMI and triglyceride level are risk factors of NAFLD, which is consistent with previous studies. Obesity itself is a kind of compensatory 
mechanism of our bodies to reserve excess energy in adipose tissue. However, fat metabolism will be impaired after exceeding the threshold level. The dysfunction of fat cells leads to insulin resistance. A large amount of fatty acids can be released and transferred to non-adipose tissue when fat catabolism is faster than synthesis. As a result, accumulation of fatty acids in the liver results in fatty liver. Hyperlipidemia, especially hypertriglyceridemia, is a risk factor of fatty liver. ${ }^{18}$ The emergence of fatty liver caused by hyperlipidemia probably results from the increased free fatty acids, and further, interferes with the combination of insulin and its receptor. Thus, reduced insulin function and insulin resistance lead to fat deposition in the liver.

In conclusion, the risk of NAFLD in young male patients with schizophrenia is apparently higher than that in the general population. Therefore, we should focus on young schizophrenia patients with fatty liver disease to avoid the risk of serious complications and even premature death. The high-risk factors of NAFLD in young male patients with schizophrenia include a combination of multiple high-dose antipsychotics, serious negative symptoms, high BMI and triglyceride level. During the therapeutic process, we should focus on the negative symptoms of patients, and use a single drug and the lowest dose possible. Besides, it is important to monitor triglyceride level and BMI regularly to remove risk factors actively, which is a simple and economic solution. Meanwhile, psychiatrists should focus on the lifestyle of patients to prevent the occurrence of fatty liver, such as controlling body weight and intake of sugar and fat, and increasing activities.

\section{Conclusion}

The risk of NAFLD in young male schizophrenia patients was significantly higher than that in the general population. Therefore, more attention should be paid to young male schizophrenia patients with fatty liver disease to avoid serious complications and premature death. The combination of high-dose antipsychotic drugs, negative symptoms, high $\mathrm{BMI}$, and hypertriglyceridemia are high-risk factors for NAFLD in young men with schizophrenia.

\section{Acknowledgment}

This work was supported by Beijing High School Youth Talent Foundation Project Plan project (no YETP0073).

\section{Disclosure}

The authors report no conflicts of interest in this work.

\section{References}

1. Newcomer JW. Second-generation (atypical) antipsychotics and metabolic effects: a comprehensive literature review. CNS Drugs. 2005; 19 (Suppl 1):1-93.

2. Vancampfort D, Knapen J, Probst M, Scheewe T, Remans S, De Hert M. A systematic review of correlates of physical activity in patients with schizophrenia. Acta Psychiatr Scand. 2012;125(5):352-362.

3. Targher G. Non-alcoholic fatty liver disease, the metabolic syndrome and the risk of cardiovascular disease: the plot thickens. Diabet Med. 2007;24(1):1-6.

4. Caldwell S, Argo C. The natural history of non-alcoholic fatty liver disease. Dig Dis. 2010;28(1):162-168.

5. Targher G, Arcaro G. Non-alcoholic fatty liver disease and increased risk of cardiovascular disease. Atherosclerosis. 2007;191(2):235-240.

6. Won BY, Park KC, Lee SH, et al. Sex difference in the association between serum homocysteine level and non-alcoholic fatty liver disease. Korean J Fam Med. 2016;37(4):242-247.

7. Chen $\mathrm{CH}$, Huang MH, Yang JC, et al. Prevalence and etiology of elevated serum alanine aminotransferase level in an adult population in Taiwan. J Gastroenterol Hepatol. 2007;22(9):1482-1489.

8. Morlán-Coarasa MJ, Arias-Loste MT, Ortiz-García de la Foz V, et al. Incidence of non-alcoholic fatty liver disease and metabolic dysfunction in first episode schizophrenia and related psychotic disorders: a 3-year prospective randomized interventional study. Psychopharmacology (Berl). 2016;233(23-24):3947-3952.

9. Fan XD, Wang XD, Yu X, et al. [World Health Organization ICD-10 Classification of Mental and Behavioral Disorder: Clinical Descriptions and Diagnostic Criteria]. Beijing: People's Health Press; 1993:72-79. Chinese.

10. Tian SL. [Drug Clinical Trials and GCP]. Beijing: Peking University Medical Press; 2003:91-101. Chinese.

11. Kay SR, Fiszbein A, Opler LA. The positive and negative syndrome scale (PANSS) for schizophrenia. Schizophr Bull. 1987;13(2):261-276.

12. Woods SW. Chlorpromazine equivalent doses for the newer atypical antipsychotics. J Clin Psychiatry. 2003;64(6):663-667.

13. Fatty liver and alcoholic liver disease group, Chinese society of Hepatology, Chinese Medical Association. [Guidelines for the diagnosis and treatment of nonalcoholic fatty liver disease]. Chin J Hepatol. 2010;18(3):163-166. Chinese.

14. Li Z, Xue J, Chen P, Yan S, Liu L. Prevalence of nonalcoholic fatty liver disease in mainland of China: a meta-analysis of published studies. J Gastroenterol Hepatol. 2014;29(1):42-51.

15. Sherif ZA, Saeed A, Ghavimi S, et al. Global epidemiology of nonalcoholic fatty liver disease and perspectives on US minority populations. Dig Dis Sci. 2016;61(5):1214-1225.

16. Ozturk K, Uygun A, Guler AK, et al. Nonalcoholic fatty liver disease is an independent risk factor for atherosclerosis in young adult men. Atherosclerosis. 2015;240(2):380-386.

17. Sim K, Su A, Fujii S, et al. Antipsychotic polypharmacy in patients with schizophrenia: a multicentre comparative study in East Asia. Br J Clin Pharmacol. 2004;58(2):178-183.

18. Slim M, Medina-Caliz I, Gonzalez-Jimenez A, et al. Hepatic safety of atypical antipsychotics: current evidence and future directions. Drug Saf. 2016;39(10):925-943.

19. Boyda HN, Procyshyn RM, Tse L, et al. Antipsychotic polypharmacy increases metabolic dysregulation in female rats. Exp Clin Psychopharmacol. 2013;21(2):164-171.

20. Correll CU, Frederickson AM, Kane JM, Manu P. Does antipsychotic polypharmacy increase the risk for metabolic syndrome? Schizophr Res. 2007;89(1-3):91-100.

21. Simpson GM, Glick ID, Weiden PJ, Romano SJ, Siu CO. Randomized controlled double-blind multicenter comparison of efficacy and tolerability of ziprasidone and olanzapine in acutely ill inpatients with schizophrenia and schizoaffective disorder. Am J Psychiatry. 2004;161(10): 1837-1847. 
22. Dwyer DS, Lu XH, Bradley RJ. Cytotoxicity of conventional and atypical antipsychotic drugs in relation to glucose metabolism. Brain Res. 2003;971(1): 31-39.

23. Dehert M, Winkel R, Eyck D, et al. Prevalence of diabetes metabolic syndrome and metabolic abnormalities in schizophrenia over the course of the illness: a cross-sectional study. Clin Pract Epidemol Ment Health. $2006 ; 2: 14$.
24. Assy N, Kaita K, Mymin D, Levy C, Rosser B, Minuk G. Fatty infiltration of liver in hyperlipidemic patients. Dig Dis Sci. 2000;45(10): 1929-1934.

\section{Publish your work in this journal}

Neuropsychiatric Disease and Treatment is an international, peerreviewed journal of clinical therapeutics and pharmacology focusing on concise rapid reporting of clinical or pre-clinical studies on a range of neuropsychiatric and neurological disorders. This journal is indexed on PubMed Central, the 'PsycINFO' database and CAS, and is the official journal of The International Neuropsychiatric Association (INA). The manuscript management system is completely online and includes a very quick and fair peer-review system, which is all easy to use. Visit http://www.dovepress.com/testimonials.php to read real quotes from published authors.

Submit your manuscript here: http://www.dovepress.com/neuropsychiatric-disease-and-treatment-journal 